\section{Biological History}

Biology in the Nineteenth Century: Problems of Form, Function and Transformation. By W. Coleman. Pp. viit 187. (Wiley Interscience: London, November 1971.) $£ 3.50$ cloth; $£ 1.75$ paper.

THE author, formerly at Johns Hopkins, now at Northwestern University, has already published a biography of Cuvier and a collection of texts on the interpretation of animal form. Like these the present work bears the mark of careful and sober scholarship. Belonging to the Wiley History of Science Series, Biology in the Nineteenth Century is aimed at the educated reader for whom it is claimed there exists no readily available work by scholars in the field.

Coleman has wisely adopted a severely selective treatment of his subject matter-nineteenth century biology -with the result that in a mere 166 pages one does get an impression of the trends within the subject and one can discern a major theme-the growing domination of many departments of the life sciences by the comparative method and the supplementation and eventual supplanting of this approach by the experimental method at the end of the century. The assumption of the comparative method, that all features of organisms are to be understood in terms of a single developmental process, led to the explanation of embryological development in terms of phylogenetic history, of which ontogeny was held to be a recapitulation, and to the placing of extant primitive cultures in a single evolutionary series. Coleman draws attention to the affinities of this method with the basic assumptions of unity and development in the writings in the German tradition of Naturphilosophie. In chapters 2-5, on the cell theory, embryology, transformism, anthropology and sociology, the comparative method is seen in action. In the last two chapters some aspects of physiology are examined, and this leads on to the reintroduction of the experimental method into descriptive subjects like embryology and variation. There is a dash of institutional history in the first and last chapters which give the lie to those who believe historians of science are oldfashioned "internalists".

The selection of material is admirable; embracing "soft" as well as "hard" sciences it does not suffer from the distortion of a commitment to the reductionist programme. One might quarrel with the author's interpretation of some points-the so-called origin of Schwann's error over the cell-forming fluid in the theory of Schleiden, the influence of Darwinism on the early anthropologists, Morgan, Lubbock and Tyler, and on the supposed abundance

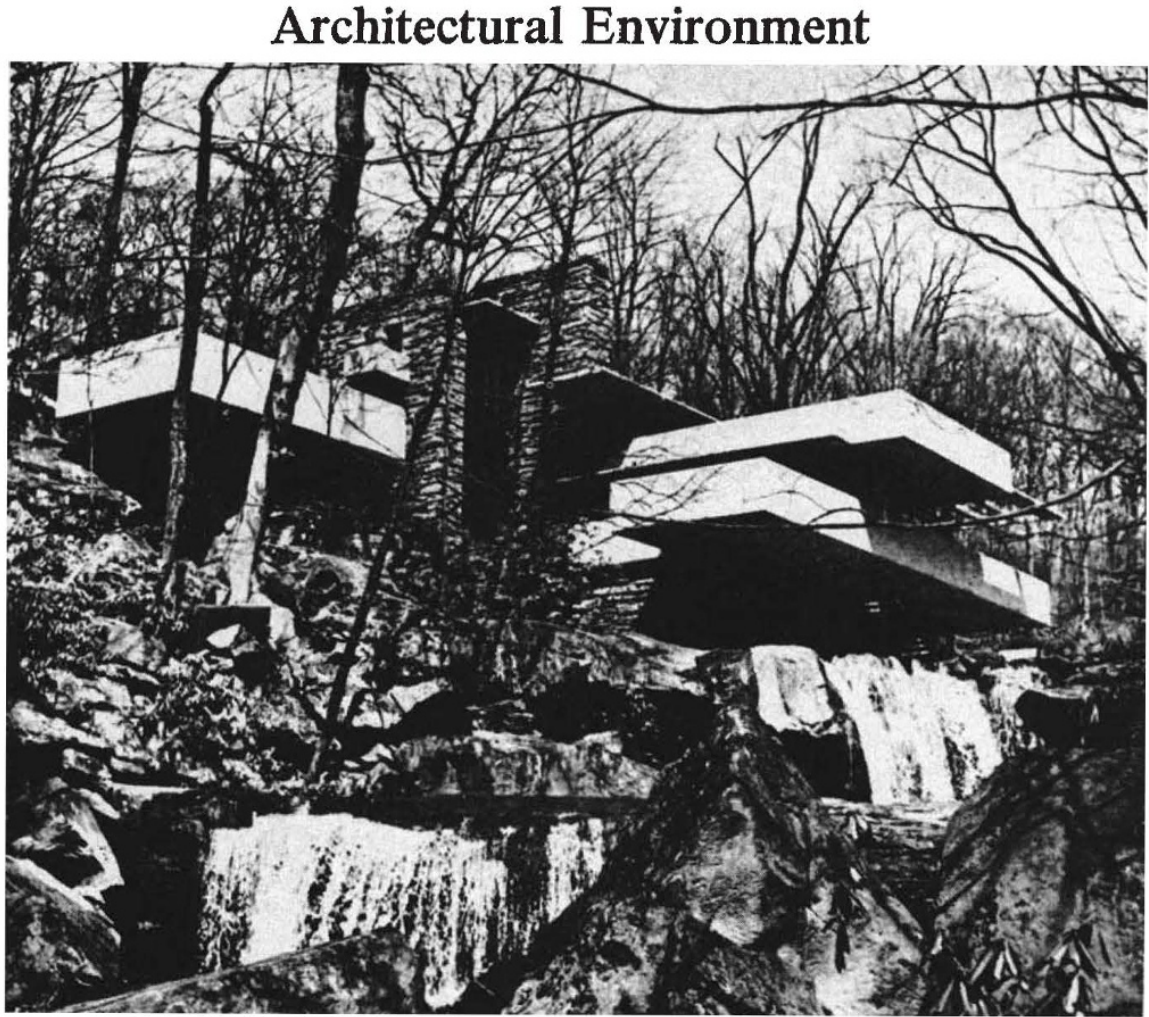

A house designed by Frank Lloyd Wright and built in Pennsylvania in 1935. This illustration, from the second edition of American Buildings, by James Marston Fitch (Houghton Mifflin, Boston, 1972; \$15.00), "tells us nothing about conditions inside where cascade is invisible, perceivable as noise, humidity chill".

of geological time for the biologists of the Darwinian era- but as an introduction to the history of nineteenth century biology this book with its excellent bibliography is unequalled. The more is the pity that Coleman has not yet developed a more natural and flowing style.

ROBERT Olby

\section{Climate}

Concepts in Climatology. By P. R. Crowe. Pp. $x x+589$. (Longman: London, October 1971.) £6.

THE atmosphere is a great heat engine whose energy source is radiation from the Sun and whose energy sink is radiation into space. The unequal distribution of the effects of these two streams of radiation generates temperature gradients and forces the air into motion on a variety of interacting scales, from that of the globe itself to scales of a few centimetres found in intense turbulence. Some of the energy is used to evaporate water at the surface of the Earth, the energy subsequently being released through condensation in clouds, and precipitation of the resulting liquid.

All these important processes are discussed by Professor Crowe in his book: the geometry of solar radiation, terrestrial radiation and its transfer, the formation of rain, jet streams, fronts and evaporation. Relevant and interest- ing statements are made about them all, although the commentary is marred at times by the author's over-anxiety to avoid technical jargon. As an example, the scattering of light may be a rather difficult concept, but to say that radiation is "tossed back" into space is hardly an improvement. This apart, the geographer for whom this book is intended will find much of interest, particularly with respect to the geographical distribution of surface variables but with the essential threedimensional structure of the atmosphere well emphasized. But Professor Crowe's intended reader is no scientist, as he states quite clearly in his introduction, where he says that . . . because ideas have evolved, ..." "a comparatively historical approach is just as appropriate as the more mathematicalphysical techniques employed by specialist meteorologists". Now I believe that the evolution of ideas is an interesting subject but meteorology, which I take to embrace climatology, would be a poor subject if there were not the occasional breakthrough which caused us to re-think our historical framework. One immediately thinks of two recent discrete jumps of this sort. Computers and numerical models of the atmosphere have shown that the motion is determinate and in response to what are basically simple laws of physics. Again, satellite pictures have shown us 